\title{
From CAARMS25 to CAARMS 2021 and Beyond: Conferences for African-American Researchers in the Mathematical Sciences William A. Massey
}

\section{Introduction}

In the early 1990s as a researcher at Bell Laboratories (then AT\&T, later Lucent Technologies, Alcatel-Lucent, and currently Nokia), I was one of many African-American mathematicians with an idea for a forum that addressed the critical issues involving African-American researchers and graduate students in the mathematical sciences. This would be a conference that would highlight current research by African-American researchers and graduate students in the mathematical sciences. It would serve to strengthen the mathematical sciences by encouraging increased participation of African-Americans, creating a community of African-American mathematicians, and providing assistance to them in cultivating their careers.

The first conference took place in 1995 at the Mathematical Sciences Research Institute (MSRI) in Berkeley, CA, hosted by the late William Thurston, who was the director of MSRI at that time. It was called the Conference for African-American Researchers in the Mathematical Sciences

William A. Massey is a professor of operations research and financial engineering at Princeton University. His email address is wmassey@princeton. edu.

Communicated by Notices Associate Editor Asamoah Nkwanta.

For permission to reprint this article, please contact:

reprint-permission@ams.org.

DOI: https://doi.org/10.1090/noti2413 or CAARMS for short. CAARMS has taken place annually from that first meeting, which is now referred to as CAARMS1. One year later, CAARMS2 was held in New Jersey at the mathematics institutions of DIMACS, Bell Laboratories, and the Institute for Advanced Study. It was cohosted by myself and the late Nathaniel Dean. As different people became involved over a 25-year consecutive string of conferences, my role was always as one of the organizers and cohosts.

This article is a chronicle of CAARMS during the recent years of 2019-2021, a time of many expected and unexpected CAARMS milestones as we celebrated our 25th anniversary in 2019. This is a conference that has all of the intimacy of a specialized workshop but the mathematical breadth of a larger conference. One legacy of CAARMS after 25 years is having over 120 CAARMS poster presenters who have since completed PhDs in the mathematical sciences. Moreover, over 50 CAARMS speakers or poster presenters have become tenured faculty in the mathematical sciences. This has led to the creation of a sustainable community of African-American mathematicians.

For each of the CAARMS events described in this article, we note the special relationships between the new CAARMS attendees and those who attended past CAARMS conferences. Under the subsection titles of "Background" we discuss some of these connections that reveal the history of the earlier ones as well as point to the 
personal-professional relationships that have helped to sustain a Black mathematical community for all these years.

We begin by reviewing the speakers and topics presented at our first day-long special session at the January 2019 Joint Mathematics Meetings in Baltimore, MD. This session was sponsored by the American Mathematical Society and informally called "CAARMS times 25 " or "CAARMS $\times 25$." We then summarize the 25th anniversary CAARMS conference, hosted by the Princeton Department of Operations Research and Financial Engineering (ORFE), held in June of 2019. Next, we reflect on the mathematical adventures of the six CAARMS attendees who participated in the School of Mathematics Summer Collaborators Program at the Institute for Advanced Study (IAS) for the month of July 2019.

The global tragedy of the COVID pandemic in 2020, coupled with the subsequent quarantines and lockdowns, brought a one-year suspension to our standard, in person CAARMS event. We discuss some of the achievements and milestones within the CAARMS community that year.

Finally the year 2021 witnessed a repurposing of CAARMS as an online conference with a new logo. The occasion also called for a new numbering system, hence the successor to CAARMS25 is now CAARMS 2021. We explore the new conference structure this inspired along with the mathematical topics that were presented. Finally, we share some of our new-found perspectives on mathematics and CAARMS events. With our renewed sense of focus and purpose, we look forward to having face-to-face meetings again in 2022 and for years to come. More details and information about the past, present, and future of CAARMS can be found at our website link caarms . princeton . edu.

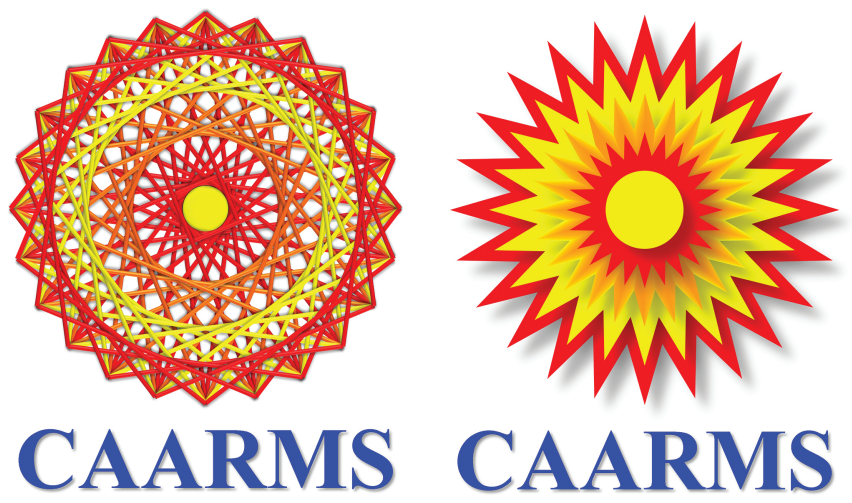

Figure 1. From the CAARMS25 logo on the left to the CAARMS 2021 logo on the right.

\section{Baltimore JMM: CAARMS $\times \mathbf{2 5}$}

To celebrate the 25th anniversary of CAARMS, we made our Joint Mathematics Meetings debut in Baltimore, MD, by running an American Mathematical Society Special Session. The CAARMS $\times 25$ speakers were:

1. "The Falconer Distance Set Problem"- Bobby Wilson, University of Washington.

2. "Queueing Theory in the Age of Technology"- Jamol Pender, Cornell University.

3. "Algorithms for Computing Tau Signatures" - Alfred G. Noël, University of Massachusetts, Boston.

4. "Numerical and Analytic Study of Dynamic Materials" - Suzanne L. Weekes, Worcester Polytechnic Institute.

5. "BPTree: an $\ell_{2}$ Heavy Hitters Algorithm Using Constant Memory" - Jelani Nelson, Harvard University.

6. "Correlation Induced by Missing Spatial Covariates: A Connection Between Variance Components Models and Kriging" - Monica C. Jackson, American University.

7. "Theory and Applications of Algebraic and Combinatorial Constructs" - Edinah K. Gnang, Johns Hopkins University.

8. "Quantum Measurement Problem" - James D. Whitfield, Dartmouth College.

9. "Dynamic Queueing Transience" - William A. Massey, Princeton University.

10. "Gabor Frames with Arbitrary Redundancy and Wilson Tight Frames in $L^{2}(\mathbb{R})$ " - Kasso Okoudjou, Tufts University.

Background.

- Bobby Wilson first spoke at CAARMS21, held at the Institute for Computational and Experimental Research Mathematics (ICERM) located in Brown University. He is a graduate of Morehouse College and was first brought to our attention by CAARMS24 speaker Ulrica Wilson.

- Jamol Pender first attended CAARMS14 in 2008 as a University of Pennsylvania graduate about to enter the Princeton ORFE PhD program, where the author became his PhD adviser. He was introduced to our conferences by Idris Stovall, who first spoke at CAARMS8 and was an inaugural attendee of CAARMS1.

- Alfred Noël first attended CAARMS2 in 1996. He was invited to CAARMS by his PhD thesis advisor, Donald King, who spoke at CAARMS4 at Rice University.

- Suzanne Weekes first spoke at CAARMS13 in 2007, held at Northeastern and the University of Massachusetts at Boston, hosted by Donald King and Alfred Noël.

- Jelani Nelson first spoke at CAARMS21 in 2015. He is now a tenured professor of computer science at the University of California at Berkeley. 
- Monica Jackson has been a longtime CAARMS organizer. She is the Deputy Provost and Dean of Faculty of American University as well as the codirector of the Summer Program in Research and Learning (SPIRAL).

- Edinah Gnang first attended CAARMS19 as a Rutgers University graduate student in 2013, when it was hosted by the University of California at San Diego, and also first spoke at CAARMS21.

- James Whitfield was an undergraduate at Morehouse College majoring in chemistry and mathematics. He received his PhD from Harvard University in Chemical Physics. He first spoke at CAARMS20 in 2014.

- William A. Massey, the author, is both a founder and a perpetual organizer of CAARMS. He is the first Black Princeton undergraduate to become a Princeton full professor. He is an inaugural Fellow of the American Mathematical Society (AMS) and a Fellow of the Institute for Operations Research and the Management Sciences (INFORMS).

- Kasso Okoudjou first attended CAARMS8 in 2002, when it was also hosted by the Princeton ORFE Department. He is one of the first CAARMS poster award winners. Moreover, his 2016 PhD student Chae Clark was an award-winning poster presenter at CAARMS21.

\section{CAARMS25 at Princeton}

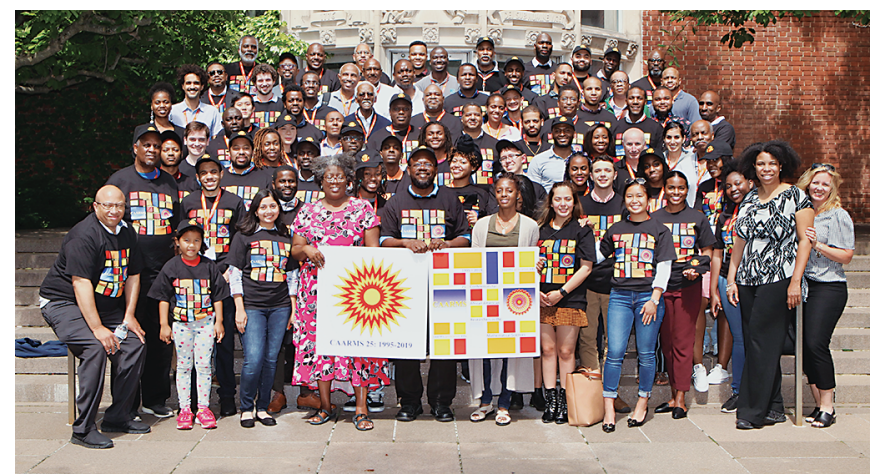

Figure 2. CAARMS25 group photo, June 2019, Princeton University.

CAARMS25 or the Twenty-Fifth Conference for AfricanAmerican Researchers in the Mathematical Sciences was held at Princeton University, with 100 students and researchers in attendance. The hosts and organizers were Gelonia Dent of Medgar Evers College, City University of New York; Monica Jackson of American University; and William A. Massey of Princeton University.

The primary CAARMS25 sponsors were the National Security Agency, the Princeton ORFE Department, and Facebook. Supporting sponsors include the Princeton University Campus Conversations on Identities (CCI), the

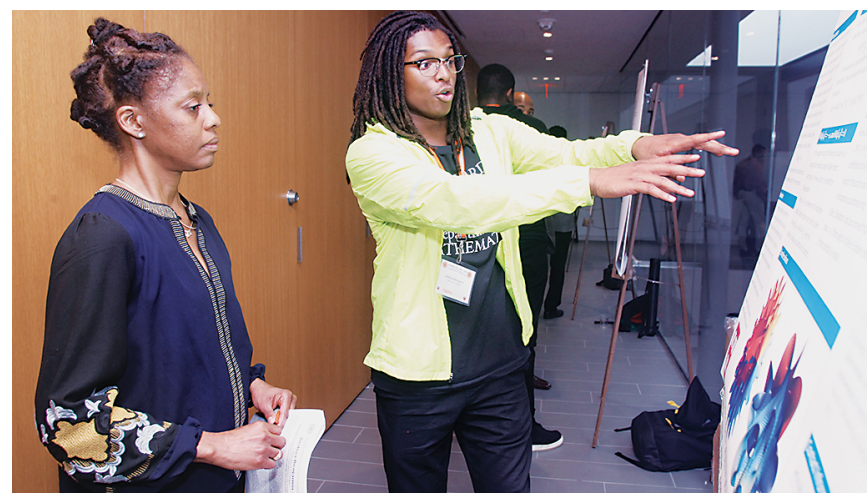

Figure 3. CAARMS25 Organizer Gelonia Dent and poster presenter Joshua Benjamin III.

Harvard University Center for Nanoscale Systems, and IvySys Technologies.

CAARMS25 began with the J. Ernest Wilkins Workshop for Future Researchers, named in memory of the outstanding mathematician J. Ernest Wilkins (1923-2011). In 1944, he was the youngest person to receive a $\mathrm{PhD}$ in mathematics from the University of Chicago. For the first ten CAARMS conferences, he was a regular attendee, a CAARMS1 inaugural speaker, and a CAARMS5 keynote speaker.

Below is the list of speakers for CAARMS25:

1. "Fractional Brownian Stochastic Differential Equations with Discontinuous Coefficients: Existence, Uniqueness \& Transportation Inequalities" - Ludovic Tangpi, Princeton University.

2. "Extremals for Morrey's Inequality" - Ryan Hynd, University of Pennsylvania.

3. "Flexible Regression Models for Dispersed Count Data" - Kimberly Sellers, Georgetown University.

4. "Recent Advances in Random Projections" - Jelani Nelson, University of California, Berkeley.

5. "Correlation Induced by Missing Spatial Covariates" Monica Jackson, American University.

6. CAARMS25 Rudy Lee Horne Jr. Speaker in Applied Mathematics: "A Weaker Notion of Convexity for Actions beyond Fluid Mechanics" - Wilfrid Gangbo, University of California, Los Angeles.

7. "CODAP: An Action Plan for Decision Making and Collaboration" - Lincoln Chandler, Chandler Decision Services.

8. "Understanding Systemic Risk at Large" - Jonathan Welburn, RAND Corporation.

9. "CAARMS Symposium on Data Science" - Organizer Otis Jennings, Mathematica with Andrea Watkins Hairston, Netflix; Karolyn Babalola, Booz Allen; and 


\section{COMMUNICATION}

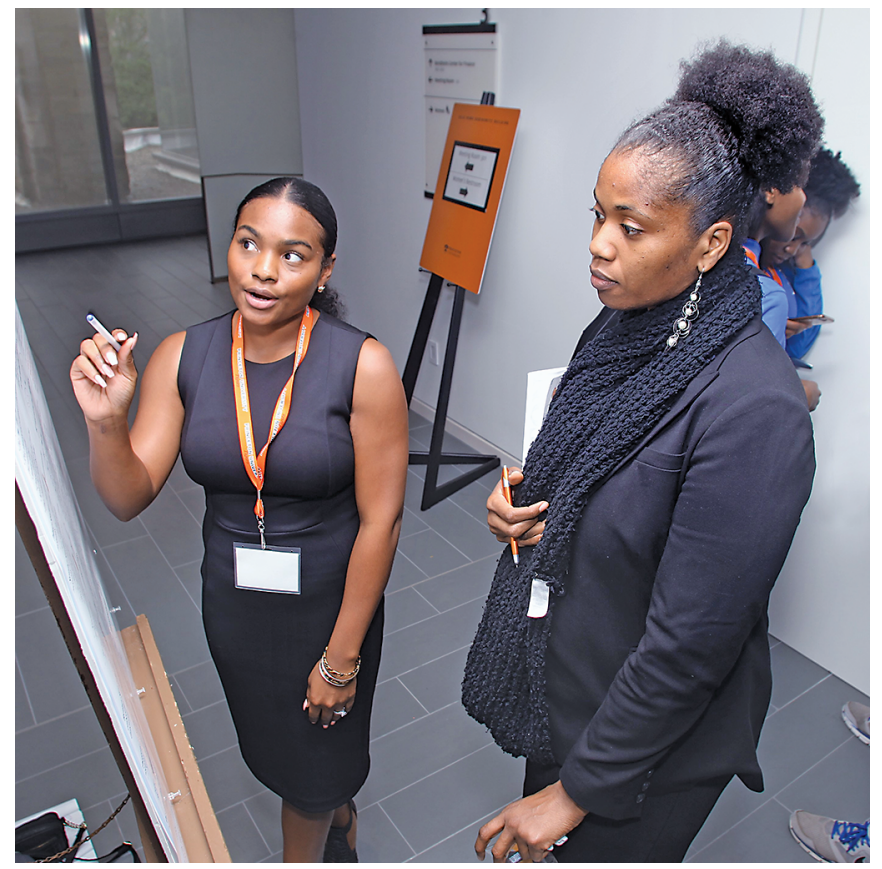

Figure 4. CAARMS25 poster presenter Jasmine Evans and symposium panelist Sydeka Watson.

Sydeaka Watson, Eli Lilly \& Company / Korelasi Data Insights.

\section{Background.}

- Ludovic Tangpi is currently an assistant professor in the Princeton Department of Operations Research and Financial Engineering. He is also an alumnus of the African Institute for Mathematical Sciences (AIMS).

- Ryan Hynd first spoke in CAARMS17, hosted by the Institute for Pure and Applied Research (IPAM) at UCLA. He was mentored as an undergraduate at Georgia Tech by CAARMS25 speaker Wilfrid Gangbo.

- Kimberly Sellers attended the inaugural CAARMS1 conference in 1995 as a graduate student. She and Monica Jackson have collaborated on joint research in statistics.

- Jelani Nelson. See Background subsection in Baltimore JMM: CAARMS $\times 25$.

- Wilfrid Gangbo was our second Rudy Lee Horne Speaker in Applied Mathematics.

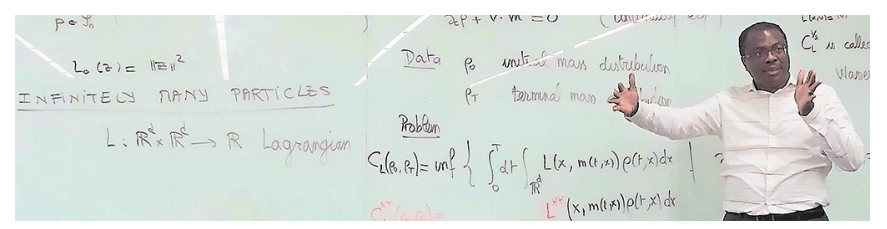

Figure 5. CAARMS25 speaker Wilfrid Gangbo.
This lecture is named after the late Rudy L. Horne (1968-2017), who was a significant presence at the first 23 CAARMS conferences before his untimely passing in 2017. In 2018 at CAARMS24, our inaugural Rudy Lee Horne speaker was Christopher Jones from the University of North Carolina at Chapel Hill and postdoctoral advisor to Rudy during the years 20032006. Wilfrid Gangbo was a postdoctoral student visiting MSRI at CAARMS1 when he first met Rudy in 1995.
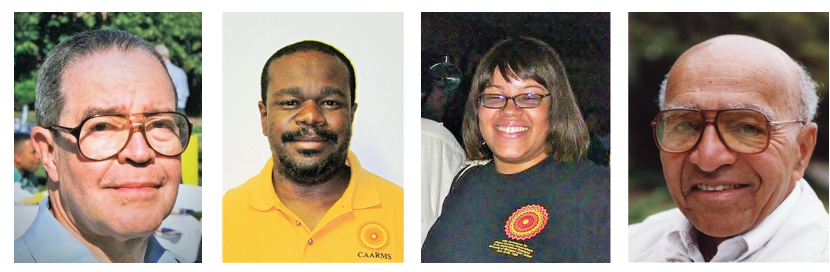

Figure 6. In memorium: left to right, J. Ernest Wilkins Jr. (1923-2011), Rudy Lee Horne Jr. (1968-2017), Angela E. Grant (1973-2010), and David H. Blackwell (1919-2010).

Rudy Horne attended CAARMS1 as a graduate student and was one of our first student poster presenters at CAARMS2. He then became one of our invited CAARMS speakers and ultimately became our CAARMS23 keynote speaker in 2017. He made a lasting impact that year as the technical advisor to the movie Hidden Figures. Many of our CAARMS photos of Rudy are featured in the memorial article, written by Della Dumbaugh and published in the Notices [1].

- Lincoln Chandler first attended CAARMS20. He spoke at CAARMS25 about his new collaborative efforts. His topic has its origins in the uniquely interdisciplinary CAARMS discussions between computer science, the data sciences, and data-driven decision sciences.

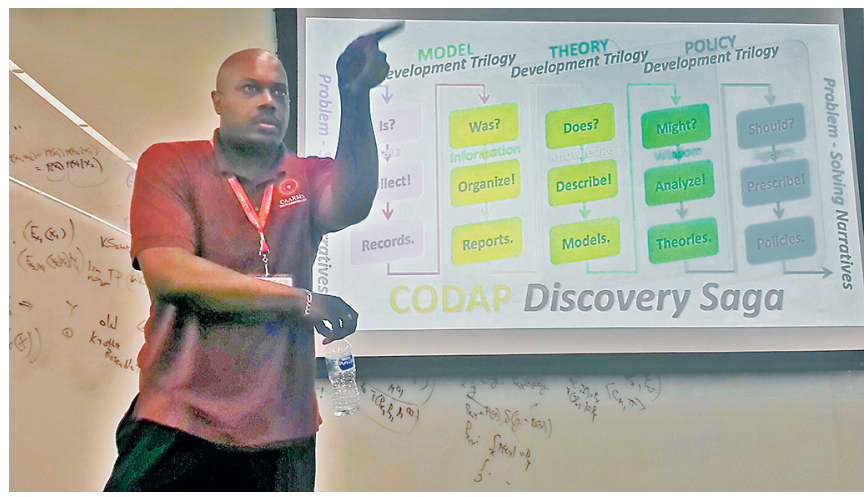

Figure 7. CAARMS25 speaker Lincoln Chandler. 
- Jonathan Welburn first attended CAARMS20 as a graduate student from the University of Wisconsin and is a former CAARMS poster award winner.

- Otis Jennings of Mathematica, who organized our CAARMS Symposium on Data Science, first attended CAARMS1 as a PhD student at Georgia Tech. The symposium began with a series of project-based case studies. Each one was highlighted by the data science contribution of the speakers.

Otis Jennings was one of the PhD thesis advisors for Andrea Hairston. Jennings' PhD lineage goes all the way back to John Robert Kline, through his PhD student Donald Flanders. Kline was the PhD advisor to three Black mathematicians. His first Black PhD student was Dudley Weldon Woodard, the second African-American to receive a PhD in mathematics. This makes Otis Jennings and Andrea Watkins the most recent Black PhDs with the closest mathematical lineage to the second African-American mathematics PhD.

CAARMS25 poster session. Many students stood before the conference audience and made brief (two minute) oral presentations about their research in our lightninground sessions. This was a prelude to the CAARMS Angela Grant Poster Session for Student Researchers held at the end of the day. At CAARMS17 in 2011, the poster session was renamed in memory of the late Dr. Angela Grant (1973-2010), a longtime active CAARMS participant and one of the first poster session award winners. In 2019 at CAARMS25, the poster event was preceded by a special tribute to Angela organized by Gelonia Dent and Monica Jackson.

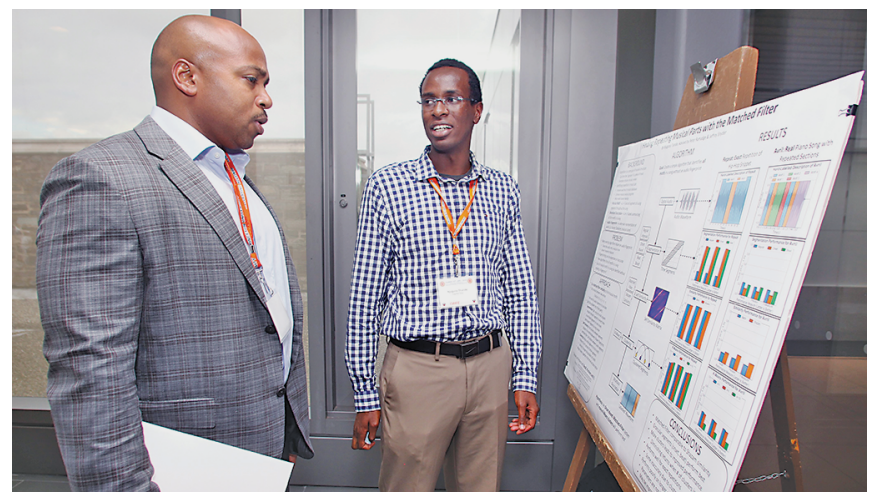

Figure 8. CAARMS25 sponsor James Debardelaben and poster presenter Njuguna Thande.

The student posters were judged by a panel of CAARMS25 participants. The award winners were:

- Best Theory Award: Joshua Benjamin III, Harvard University (second from the right in Figure 9), for
"Existence of Kähler-Einstein Metrics on Compact Manifolds."

- Best Modelling Award: Nicholas A. Johnson, Princeton University (on the far right of Figure 9), for "General Purpose Monte Carlo Optimisation Engine in $\mathrm{R}$ with Acceptance Ratio Annealing and Replica Exchange."

- Best Presentation Style Award: Jasmine Evans, Morgan State University (center of Figure 9), for "RNA Combinatorics and Prediction of microRNAs in Cancer."

- Best Algorithm Award: Mourchid Adegbindin, École Polytechnique de Montréal (second from the left of Figure 9), for "A New Efficient Algorithm for the Vertex Coloring Problem."

- Outstanding Undergraduate Award: Njuguna Thande, Princeton University (in Figure 8), for "Finding Repeating Musical Parts with the Matched Filter."

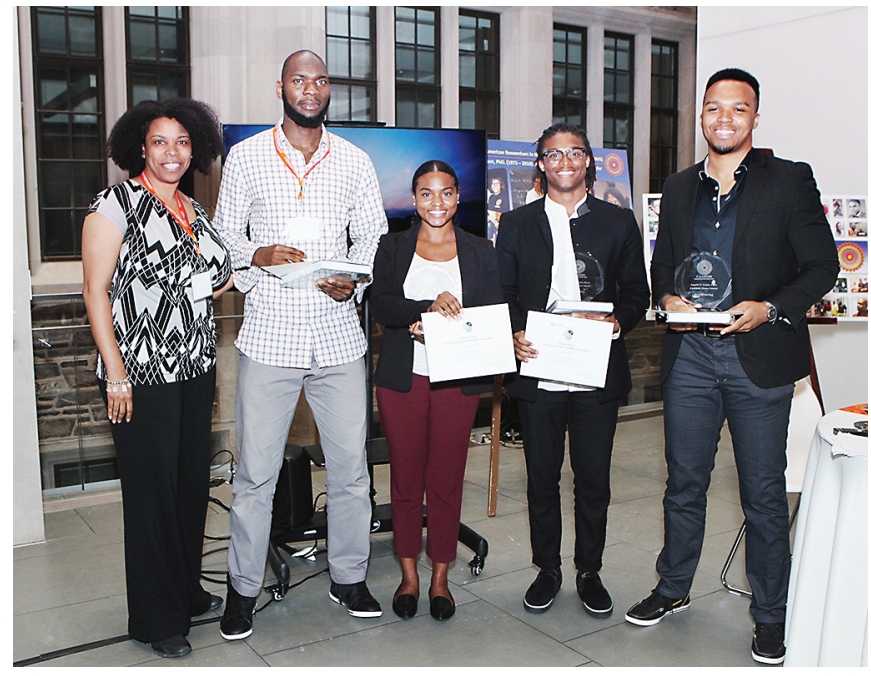

Figure 9. CAARMS25 speaker Kimberly Sellers (left) with the poster award winners.

CAARMS25 concluded with the David Blackwell Workshop for Future Research, named in memory of the late Professor David Blackwell (1919-2010), a leading pioneer in mathematical statistics and the most accomplished African-American mathematician of the 20th century. This is a forum where the CAARMS poster winners give 20minute lectures about their work.

In September 2019, our CAARMS poster winner Nicholas Johnson received the Class of 1939 Princeton Scholar Award. This is awarded each year to "the Princeton undergraduate who, at the end of junior year, has achieved the highest academic standing for all preceding college work at the University." 


\section{COMMUNICATION}

In May of 2020, he was selected to be the Princeton valedictorian for the class of 2020. This made him the first Black valedictorian in the 274-year history of Princeton as well as the first ORFE major valedictorian in the 20-year history of the department.

In December of 2020, our CAARMS25 poster winner Jasmine Evans became the first African-American mathematics PhD graduate of Morgan State University. Her PhD advisor was a CAARMS1 inaugural attendee, Asamoah Nkwanta, chair of the Morgan State University Mathematics Department.

\section{IAS Summer Collaborators}

Working with the Institute for Advanced Study, six CAARMS participants participated in the IAS School of Mathematics Summer Collaborators Program. Organizer William A. Massey, together with Robert Hampshire, Alfred Noël, and Jamol Pender, spent the entire month of July at IAS working on new research projects centered around the theme of the Spectral Dynamic Analysis of Stochastic Service Networks. They were joined by CAARMS25 attendees Paul Fomenky, a former PhD student of Alfred Noël, and Princeton ORFE graduate student Emmanuel Ekwedike.

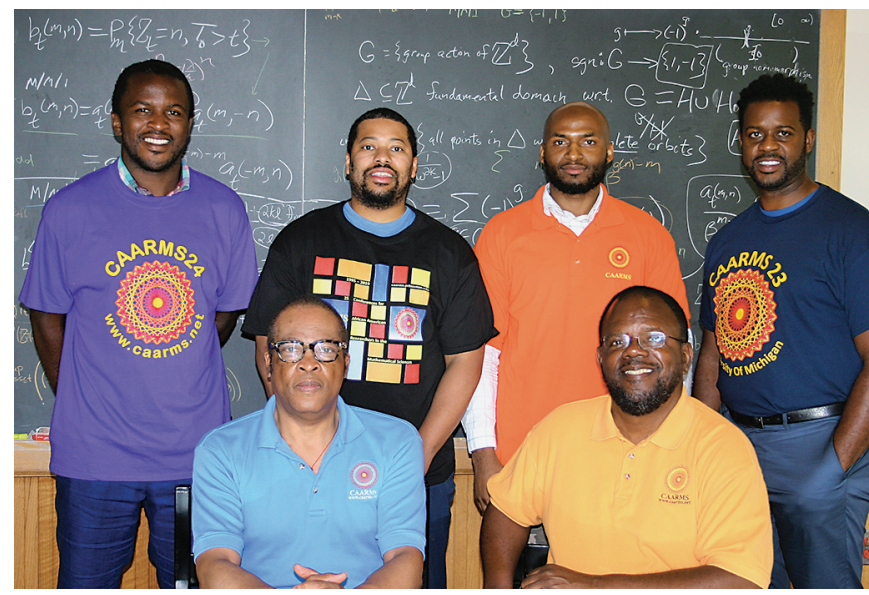

Figure 10. IAS School of Mathematics Summer Collaborators Program, July 2019.

The synergy of the IAS Summer Collaborators program led to the final chapter of the PhD dissertation for CAARMS25 attendee Emmanuel Ekwedike, who successfully defended his thesis in August of 2020.

\section{CAARMS 2021 Online}

As mentioned in the Introduction, because of the global pandemic, there was no CAARMS in 2020 and CAARMS 2021 was reimagined as an online conference. The lectures were organized around four major themes: Machine
Learning and Statistics, Data Driven Decision Making, Quantum Materials and Quantum Computation, and finally Transportation and Infrastructure.

The speakers for CAARMS 2021 were:

1. "Estimating Causal Peer Influence in Homophilous Social Networks by Inferring Latent Locations" - Edward McFowland III, Harvard Business School.

2. "Leveraging Dataset Structure for Neural Network Prediction" - Christine Blanchette-Allen, Princeton University.

3. "Coming to Grips with the Reality of Data Science - It's People All the Way Down - Round 2" - Vukosi Marivate, University of Pretoria.

4. "Comparing a Large Scale Machine Learning System to Academic ML" - Frederick Campbell, Microsoft.

5. "Beyond Safety Drivers: Staffing a Teleoperations System for Autonomous Vehicles" - Jamol Pender, Cornell University.

6. "Internet Measurement for the Prevention and Detection of Internet Surveillance" - Laura Roberts, Princeton University.

7. "The Development of COVID-19 Vaccine Equity Index Decision Making Using Conditional Probability with Future Applications" - Stephen Lockhart MD, former Chief Medical Officer for Sutter Health.

8. "How to Build a Quantum Computer with Topological Materials" - Jacob Gayles, University of South Florida.

9. "Quantum Computing: What's the Hype?" - James Whitfield, Dartmouth College.

10. "Research, Data, and Policy at the Department of Transportation" - Robert Hampshire, United States Department of Transportation.

The CAARMS 2021 events were scheduled to accommodate the geographical spread of our participants. In addition to CAARMS participants on the East Coast, we had West Coast registrants in the San Francisco Bay Area, like N. Duru Ahanotu, Harry Bims, and Jean Borgella, and in the Seattle area, like our speaker Frederick Campbell. In addition, we had Leonce Mekinda in Hamburg, Germany, Patrick Tenga in Alexandria, Egypt, and our speaker Vukosi Marivate in Pretoria, South Africa, all in times zones six hours later than eastern standard time.

Background.

- Edward McFowland III was invited by Robert Hampshire to attend CAARMS17 as a graduate student poster presenter.

- Christine Blanchette-Allen has a PhD in Computer Science from the University of Pennsylvania and currently has a postdoctoral position in the Princeton Department of Mechanical and Aerospace Engineering. 
She will become an assistant professor in the same department by the Fall of 2022.

- Vukosi Marivate is currently the UP ABSA Chair of Data Science at the University of Pretoria and CoFounder of Deep Learning Indaba. Edinah Gnang is one of his graduate student classmates from Rutgers University. He was a CAARMS20 poster presenter back in 2014.

- Jamol Pender. See Background subsection in Baltimore JMM: CAARMS $\times 25$.

- Laura Roberts became the second Black person to receive a Computer Science PhD from Princeton University in 2021. The first is Corey Toler-Franklin, who presented her work at CAARMS17 in 2011 at IPAM.

- Stephen Lockhart MD also has a PhD in Biostatistics. Along with the author and Edward McFowland III, he hails from Saint Louis, Missouri, where he graduated from Washington University as a Rhodes scholar.

- Jacob Gayles also attended CAARMS17 as a graduate student poster presenter. Gayles and James Whitfield jointly gave the Rudy Lee Horne Jr. Applied Mathematics Lectures for CAARMS 2021. The day before their main lectures, they both gave hour-long tutorials on quantum mechanics.

- Robert Hampshire is currently serving as the Deputy Assistant Secretary for Research and Technology and Chief Science Officer at the United States Department of Transportation under the Biden Administration. The author was his PhD adviser at the Princeton ORFE Department. He was first a CAARMS7 graduate student poster presenter at Duke University in 2001.

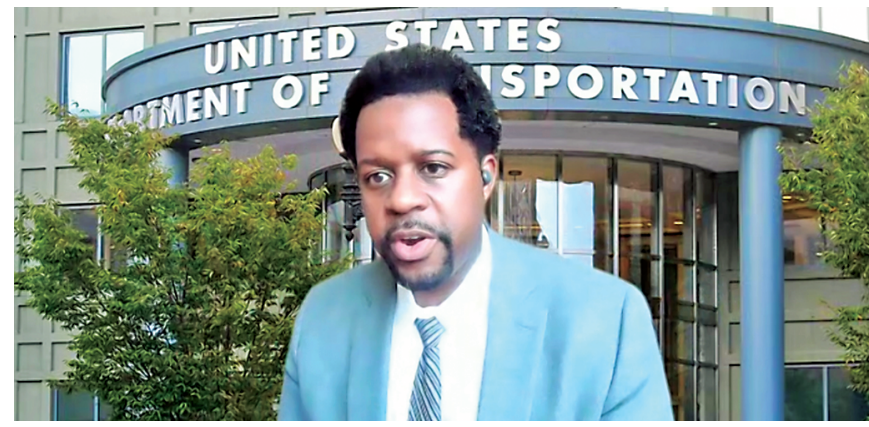

Figure 11. CAARMS 2021 Speaker Robert C. Hampshire, Deputy Assistant Secretary for Research and Technology and Chief Science Officer at the US Department of Transportation.

CAARMS 2021 Workshop Projects. Along with the quantum tutorials, CAARMS 2021 included two workshop presentations:

1. "CAARMS Data from CSV to 3D" - Orville Dillon Jr., New Jersey Institute of Technology.
2. "The Hidden Equations behind the Hidden Figures" Elisha Emadasu, Medgar Evers College, City University of New York.

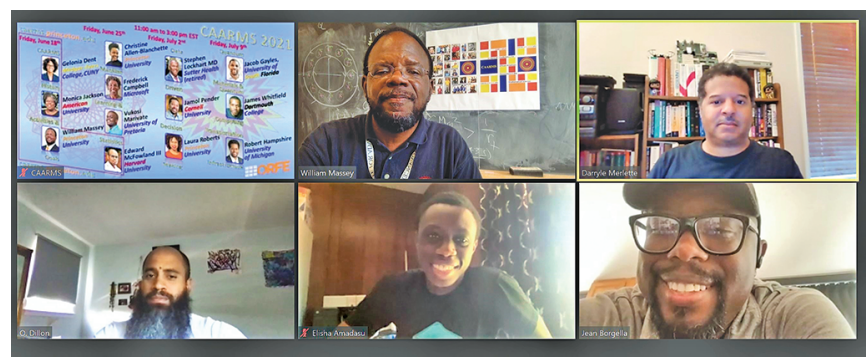

Figure 12. Online CAARMS 2021 Workshop.

The first Workshop Project was to create an infographic display of mathematical interests of CAARMS 2021 registrants. Here, we borrow from the collaborative culture of computer science to organize this project as a workflow. We begin with Mathematics Subject Classification System (MSC2020) data collected on the CAARMS 2021 registration form, designed by ORFE Information Technology Manager Michael Bino with Drupal. This information was sent to ORFE Events Coordinator/Communications Administrator Tabitha Mischler. She forwarded a resulting Microsoft Excel speadsheet to CAARMS organizer William Massey. He exported the data as a graphic image and forwarded it CAARMS Workshop participant Darryle Merlette. He applied Unix shell script commands to clean the data and then handed it off to CAARMS Workshop participant and presenter Orville Dillon Jr., who used Python to render the 3-dimensional color plot of the data in Figure 13.

In the figure, 2-digit MSC2020 numbers are displayed in the $x y$-plane using a 10-by-10 array where the first digit is represented by the row and the second digit is represented by the column. For example, the number 60 for Probability and Stochastic Processes corresponds to the 6th row and the 0th column of the array. The $z$-axis gives the number of CAARMS registrants selecting a given MSC2020 number as a subject of interest. To make it easier to see broad areas of mathematics, we use the following color scheme:

- Red: Algebra, Discrete Mathematics, and Logic

- Orange: Analysis, Differential Equations, Probability, and Stochastic Processes

- Yellow: Geometry and Topology

- Green: Mathematics of Nature and Science

- Blue: Mathematics of Technology, Computer Science, Operations Research, and Statistics

- Violet: Biography, Education, and History of Mathematics. 


\section{COMMUNICATION}

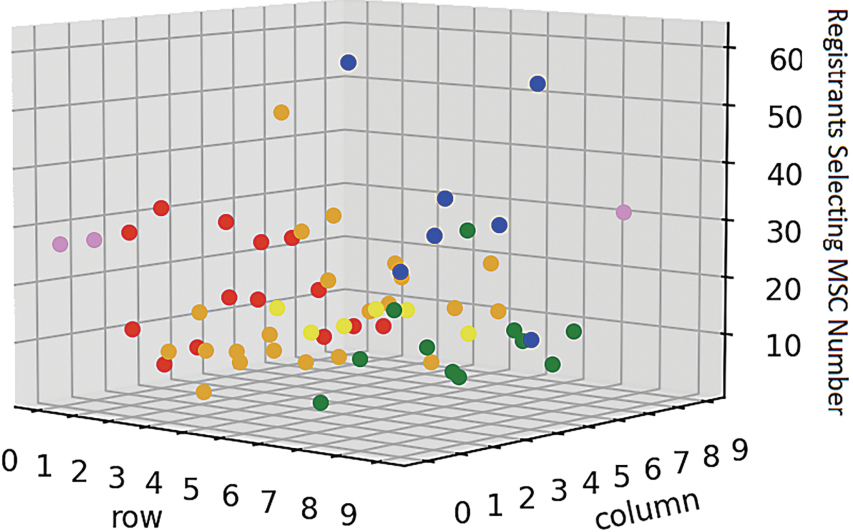

Figure 13. 3D spectral decomposition of the CAARMS 2021 registrants' mathematical interests.

This representation shows us that the most popular CAARMS 2021 subject was statistics, index \#62 with 60 out of 150 CAARMS 2021 registrants. This is followed by a tie for probability \& stochastic processes, index \#62 with 53 registrants, along with computer science, index \#68 with 53 registrants. In fact, every 2-digit MSC2020 entry was selected by at least one CAARMS 2021 registrant. This speaks to the breadth of interests of all the attendees.

The second Workshop Project reviewed the numerical solution techniques (like the Forward Euler method) suggested by Katherine Johnson to solve the differential equations for the two-body problem; see Shetterly [2]. CAARMS Workshop participant Elisha Emadasu presented this material. He was invited to attend CAARMS 2021 while he was enrolled in a mathematics class at Medgar Evers College taught by CAARMS organizer Gelonia Dent.

\section{Summary and the Future}

Building Black communities to create mathematics. This is the CAARMS mission. Unpack the word research and you are talking about "creating mathematics." CAARMS gives students a sense of the larger world of mathematical creation and how all of the disparate parts fit together. This enables them to support each other professionally.

The CAARMS perspective on making mathematics more diverse is by increasing the number of African-American researchers in the mathematical sciences. Increasing these numbers is the fastest way to diversify contributions to mathematics. Along with adding to the body of mathematics, academic researchers produce the next generation of PhDs. Hence increasing the number of African-American academic researchers adds a strong multiplier effect to increasing the number of African-American PhDs.
Think outside the hypercube. This has been our closing tagline at many CAARMS conferences, where we stress the importance of creative thinking for contributing to mathematics.

Look beyond the silos. Being from an underrepresented group makes you highly sensitive to the folly of isolating yourself into a narrow professional silo. Real life is actually interdisciplinary and we have found that CAARMS attendees walk away more attuned to how various types of mathematical techniques relate to specific application domains.

In closing, readers can learn more details and information about CAARMS at the website caarms.princeton .edu.

ACKNOWLEDGMENT. This paper was written during a sabbatical from Princeton University while being a Member of the School of Mathematics at the Institute for Advanced Study during the year 2020-2021.

\section{References}

[1] Della Dumbaugh, Rudy Lee Horne: the hidden figure of Hidden Figures, 1968-2017, Notices Amer. Math. Soc. 66 (2019), no. 2, 202-210. MR3840123

[2] Margot Lee Shetterly, Hidden figures: The American dream and the untold story of the Black women mathematicians who helped win the space race, HarperCollins Nordic, 2017.

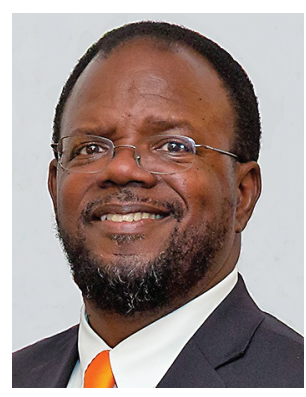

William A. Massey

Credits

Figures 1-5, 6 (photos of J. Ernest Wilkins, Jr., Rudy Lee Horne, Jr., and Angela E. Grant), 7-9, and 11-13 are courtesy of William A. Massey.

Figure 6 photo of David H. Blackwell is courtesy of George A. Bergman. It is under the Creative Commons AttributionShare Alike 4.0 International license.

Figure 10 is courtesy of the Institute for Advanced Study.

Photo of William A. Massey is courtesy of Princeton University. 